\title{
Novel free boundary equilibrium and transport solver with theory-based models and its validation against ASDEX Upgrade current ramp scenarios
}

\author{
E. Fable, C. Angioni, F. J. Casson, D. Told, A. A. Ivanov ${ }^{1}$, \\ F. Jenko, R. M. McDermott, S. Yu. Medvedev ${ }^{1}$, G. V. \\ Pereverzev $\ddagger$ F. Ryter, W. Treutterer, E. Viezzer, \\ and the ASDEX Upgrade Team \\ Max-Planck-Institut für Plasmaphysik, EURATOM Association, 85748 Garching, \\ Germany \\ ${ }^{1}$ Keldysh Institute, Russian Academy of Sciences, Moscow, Russia \\ E-mail: emiliano.fable@ipp.mpg.de
}

\begin{abstract}
Tokamak scenario development requires understanding of the properties that determine the kinetic profiles in non-steady plasma phases, and of self-consistent evolution of the magnetic equilibrium. Current ramps are of particular interest since many transport-relevant parameters explore a large range of values and their impact on transport mechanisms has to be assessed. To this purpose a novel full-discharge modeling tool has been developed, which couples the transport code ASTRA [1] and the free boundary equilibrium code SPIDER [2], utilizing a specifically designed coupling scheme. The current ramp-up phase can be accurately and reliably simulated using this scheme, where a plasma shape, position, and current controller is applied, which mimics the one of ASDEX Upgrade. Transport of energy is provided by theory-based models (e.g. TGLF [3]). A recipe based on edge-relevant parameters [4] is proposed to resolve the low current phase of the current ramps, where the impact of the safety factor on microinstabilities could make quasi-linear approaches questionable in the plasma outer region. Current ramp scenarios, selected from ASDEX Upgrade discharges, are then simulated to validate both the coupling with the free-boundary evolution and the prediction of profiles. Analysis of the underlying transport mechanisms is presented, to clarify the possible physics origin of the observed L-mode empirical energy confinement scaling. The role of toroidal micro-instabilities (ITG, TEM) and of non-linear effects is discussed.
\end{abstract}




\section{Introduction}

Full-discharge modeling of tokamak plasma scenarios is recognized as a necessity to assess viability of the considered scenario, from current ramp-up to flat-top to rampdown, in terms of controller requirements through the impact of plasma transport on the kinetic and magnetic profiles [5, 6, 7, 8, 9, 10]. These kind of simulations are becoming of importance for ITER operation design [11], and full-discharge modeling of ITER has already started [12]. Previous work at ASDEX Upgrade (AUG) on current ramp scenarios has been focused on transport properties and validation of transport models $[13,14,15]$.

It would be desirable to have a tool that could compute self-consistent profiles from magnetic axis to machine wall at all time scales, however the computational time takes its toll. Focusing on the core plasma, one could "ignore" first the SOL physics and perform 1.5D modeling where the plasma is coupled to the external environment through mutual inductances and eddy currents. Moreover the Alfvén and modes with $n>0$ dynamics are ruled out, which excludes part of the phenomenology related to asymmetric VDEs for example. In the same way turbulence is treated as istantaneous and computed with fast, quasi-linear codes.

The mentioned assumptions are behind the numerical tool developed to perform this work, namely the coupling of the ASTRA code with the free-boundary evolution code SPIDER and a quasi-linear turbulent transport model. The motivation for developing this package is twofold: 1) to have a flexible and modular transport-equilibrium package; 2) to validate the physics model implemented in the code against experimental scenarios for predictive capabilities on present and future devices (e.g. ITER, DEMO).

Three key questions are assessed in this work. 1) equilibrium problem: is the tool able to reproduce experimental evolution of relevant parameters of AUG cases with prescribed kinetic profiles $(\mathrm{T}, \mathrm{n})$ ? 2) transport-equilibrium problem: is the quasilinear transport model able to predict $T_{\mathrm{e}}$ evolution ? 3) turbulent transport problem: is the physics of core-edge transport present in the quasi-linear transport model sufficient to predict the $T_{\mathrm{e}}$ profile. If not, with help from non-linear gyro-kinetic simulations performed with GENE and GKW codes [16, 17], the missing ingredients are searched for.

The present work is divided in three main sections: section 2 presents the validation of the free-boundary transport model on current ramp-up and ramp-down scenarios in AUG. Section 3 presents the gyro-kinetic analysis of a plasma current scan. Section 4 draws the conclusions.

\section{Validation of the free-boundary-transport model on AUG current ramp scenarios}

The coupled ASTRA-SPIDER package allows one to evolve the 1D kinetic profiles inside the plasma boundary which also evolves in time due to the coupling with the 
external coils. The ability to perform controlled (in position and shape) scenarios has been previously demonstrated, with good agreement with respect to the measured parameters (position, shape, and coil currents) [18]. Now two new features have been added: plasma current control, and the quasi-linear turbulence-driven transport model TGLF [3]. Details on the AUG controller for current, position and shape can be found in Ref. [19].

The TGLF model has been shown to satisfactorily predict core profiles of several DIII-D discharges [20]. The same has been recently reported also for several AUG stationary cases [21]. However it has been also found to under-estimate transport in 'pathological' cases, in particular as the safety factor $q$ is increased [22]. Since here the purpose is to model current ramps, where $q$ explores a large range of values, say $4 \lesssim q_{95} \lesssim 16$, to prevent this a "non-linear enhancement" is applied. The enhancement is an addition of constant head diffusivities in the radial region defined by the condition $\rho_{\mathrm{n}} \geq \rho_{\mathrm{n}}^{\text {crit }}\left(\rho_{n}=\sqrt{\Phi / \Phi_{\mathrm{b}}}\right.$ with $\Phi$ the toroidal magnetic flux $)$. The increment is $\propto R / a q_{95} / \sqrt{k_{\mathrm{b}}}$, where the constant in front of this factor is $2 \mathrm{~m}^{2} / \mathrm{s}$ (this scaling is a very simple way to enforce the current scaling, however refinement of this choice is planned as future work). The value of $\rho_{\mathrm{n}}^{\text {crit }}$ is the location where $\max (\hat{\mu}, \hat{\beta}, \hat{C})=1$. The three parameters $\hat{\mu}, \hat{\beta}, \hat{C}$ definitions can be found in Ref. [4]. The physical argument for choosing these parameters in defining the enhancement region is the following: when either of them becomes $O(1)$, passing electrons dynamics becomes strongly non-adiabatic, and additional non-linear drift-wave physics takes place, contributing to turbulent energy transfer and pumping the transport fluxes [4]. It will be demonstrated in the next sections that the wording "non-linear enhancement" is actually very appropriate. Notice that the non-linear enhancement is applied equally to both the ions and electron heat transport channels, although future investigations shall give a more quantitative description on how to apply this enhancement in the two channels, as well as its qualitative dependencies.

The transport model is then completed by adding neoclassical transport from NCLASS [23], and the current conductivity from Ref. [24]. The boundary conditions for $T_{\mathrm{e}}, T_{\mathrm{i}}$ are fixed at $\rho_{\mathrm{n}}=0.95$, following the experimental values at that location.

As regards the free-boundary solver, circuit parameters and the controller scheme are taken from AUG data and schematics. Notice that however here the controller is simplified, as in AUG there are many inter-connected switches that have not been implemented yet in the code. Controlled quantities must follow target values that also come from AUG data. For the control of the plasma vertical position in particular, the focus is on the active control coils (called "ICoIo") and on the passive stabilizer placed inside the vacuum vessel (called "IPSLo"). A model for the sawtooth crash has been implemented [25]. Auxiliary ECRH heating is modeled with the TORBEAM code [26] inside the transport simulation.

Input data are taken either from ECE for $T_{\mathrm{e}}$, CXRS for $T_{\mathrm{i}}$, and Thomson scattering or IDA [27] for $n_{\mathrm{e}}$. Notice that in the modeling the density profile will always be prescribed and not will not be modeled. Initial equilibria used as guess for the free- 
boundary code are taken from the equilibrium reconstruction code CLISTE [28]. The effective charge $Z_{\text {eff }}$ is obtained from IDZ [29] methodology. The impurity assumed to cause the observed effective charge is oxygen. The plasma core radiation profile is obtained from the core part of the measured radiation. It is assumed it is produced by $97 \%$ of $\mathrm{O}$ and $3 \%$ of $\mathrm{W}$ contribution to $Z_{\text {eff }}$. This results in $\sim 10^{-4}-10^{-5} \mathrm{~W}$ concentration, in line with what is typically observed during current ramps in AUG.

Numerical convergence of the obtained results has been checked on one case by either: reducing the time step, reducing the tolerance on the equilibrium solution, and increasing the numnber of calls to TGLF in a confinement time. These tests have resulted in negligible change in the time evolution, thus giving confidence that the numerical parameters chosen to model all cases should not impact on the result, while keeping the computational time affordable.

\subsection{Current ramp-up scenarios}

Ohmic current ramp-up \#26328 and ECRH-heated ramp-up \#26904 are chosen to carry out the validation exercise. Both are ran at $B_{\mathrm{T}}=2.5 \mathrm{~T}$. Discharge \#26904 has $\approx 0.65 \mathrm{MW}$ of additional central ECRH applied from $t=0.3 \mathrm{~s}$ up to the end of the current ramp. In both cases the current ramp-up ends around $t \approx 1.1 \mathrm{~s}$, with a ramp-rate $\approx 0.7 \mathrm{MA} / \mathrm{s}$. More details on these scenarios can be found in Ref. [14].

Two sets of simulations are carried out: one with prescribed kinetic profiles (only $\psi$ is evolved through current diffusion), and the second with evolving $T_{\mathrm{e}}, T_{\mathrm{i}}$ using TGLF plus the transport enhancement described previously. The results are presented in figure 1 for \#26328 and figure 2 for \#26904.

The plots show that the free-boundary solver is able to quantitatively reproduce the global evolution of plasma parameters. The agreement is very good, particularly for the $I_{\mathrm{OH}}$ time trace, meaning that the code can correctly compute the plasma current evolution which, due to the mixed boundary condition on $\psi$, is not an obvious task numerically. The simulations performed with TGLF+non-linear enhancement agree very well with the simulations performed at fixed kinetic profiles. On the other hand, if no enhancement is used, it is observed that the $\mathrm{OH}$ current slope is much shallower, due to an overestimate of edge $T_{\mathrm{e}}$. This point will be discussed further in next sections.

\subsection{Current ramp-down scenarios}

Two Ohmic current ramp-down \#29643, \#29644 are simulated. The ramp-down rate is respectively $\approx-0.75 \mathrm{MA} / \mathrm{s}$ and $\approx-0.33 \mathrm{MA} / \mathrm{s}$, while all other discharge parameters are the same. In figure 3 and figure 4 respectively the results of the simulations are presented, with the same time traces plots as in figure 1.

The results show very good agreement for the plasma current, the $I_{\mathrm{OH}}$ current evolution, and the resultant loop voltage $V_{\text {loop }}$, while some discrepancy is observed on geometrical parameters and the active/passive position stabilizing coils. The reason for the latter discrepancy could be related to the behaviour of the outer control coils, which 

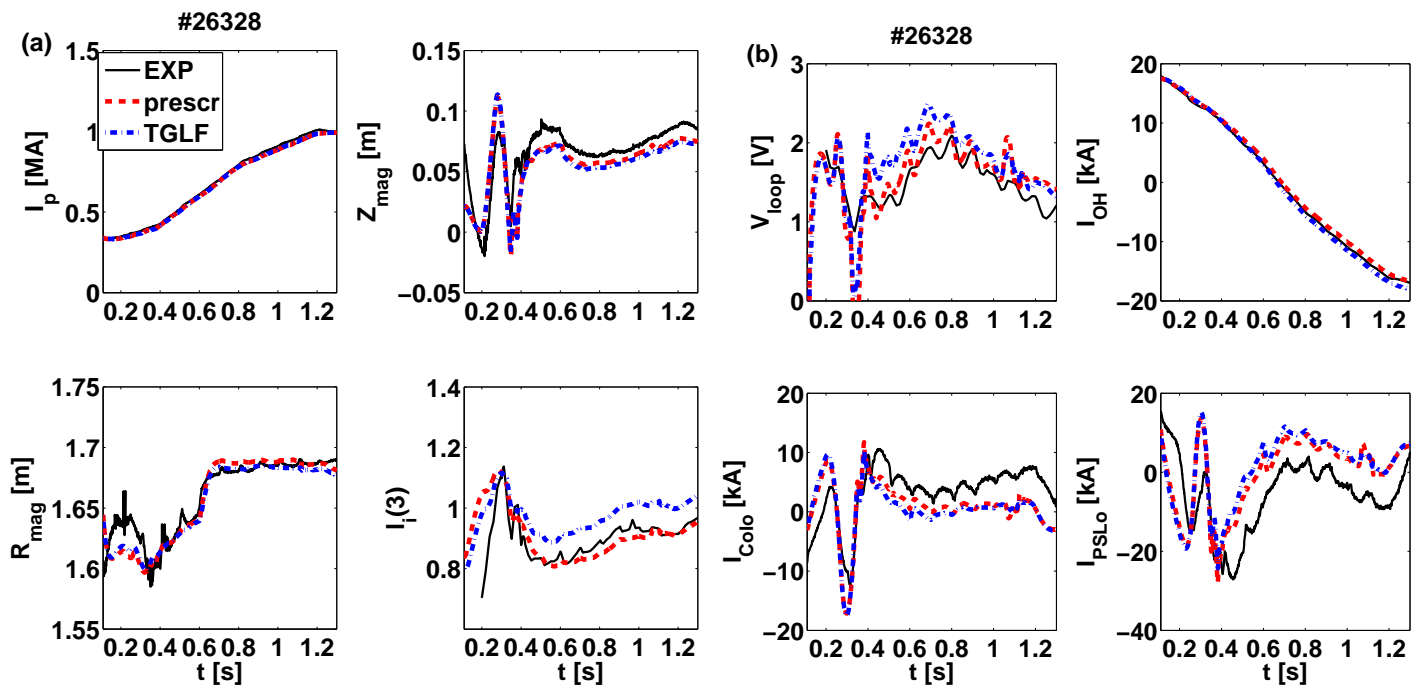

Figure 1. For ramp-up \#26328: a) Time traces of plasma current $I_{\mathrm{p}}$ [MA] (topleft), magnetic axis vertical position $Z_{\text {mag }}$ (top-right) and major radius $R_{\text {mag }}$ [m] (bottom-left), internal inductance $l_{\mathrm{i}}(3)$ (bottom-right); b) time traces of boundary loop voltage $V_{\text {loop }}[\mathrm{V}]$ (top-left), Ohmic transformer current $I_{\mathrm{OH}}[\mathrm{kA}]$ (top-right), active control coil current $I_{\text {CoIo }}[\mathrm{kA}]$ (bottom-right), and passive stabilizer current $I_{\mathrm{PSLo}}[\mathrm{kA}]$ (bottom-left). For all plots the solid black curve is from measurements and CLISTE reconstruction ('EXP'), the dashed red curve is from the simulation with fixed kinetic profiles ('prescr'), and the dot-dahsed blue curve is from simulations including the TGLF transport model for $T_{\mathrm{e}}, T_{\mathrm{i}}$ ('TGLF').
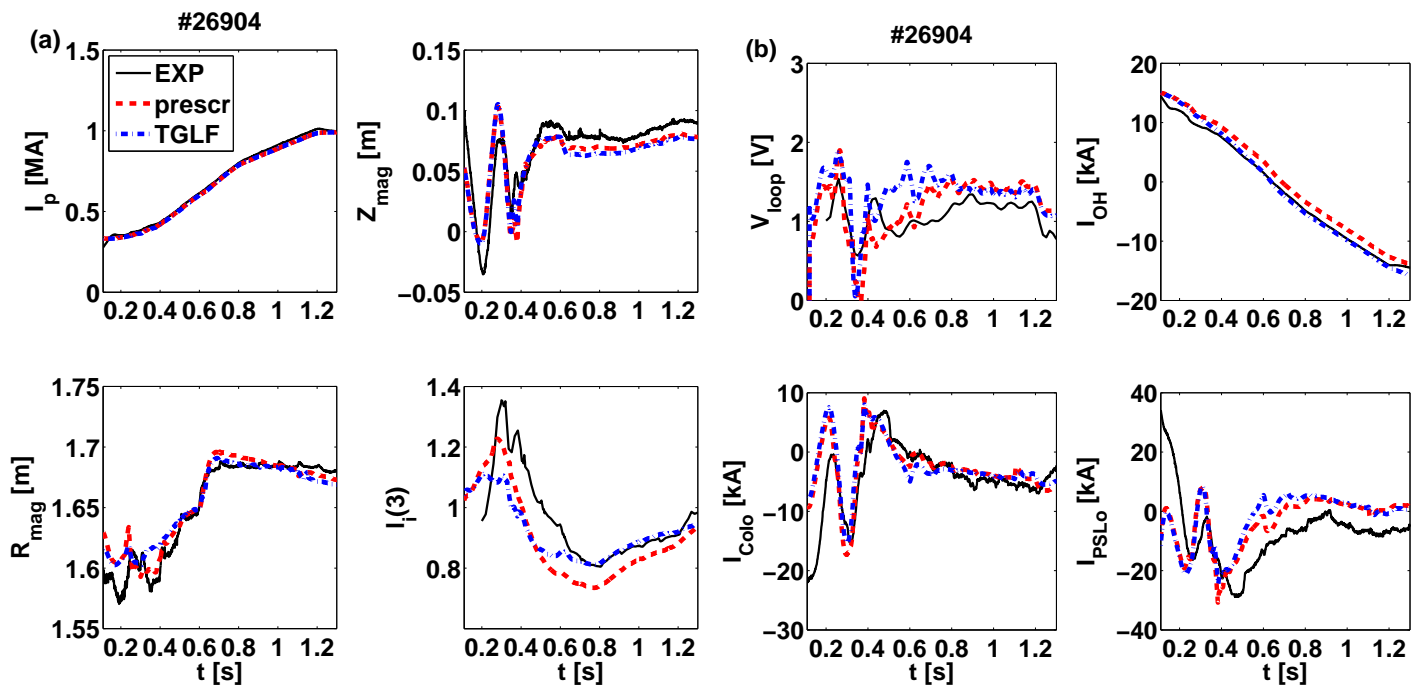

Figure 2. Same plot as in figure 1, but for ramp-up \#26904. 

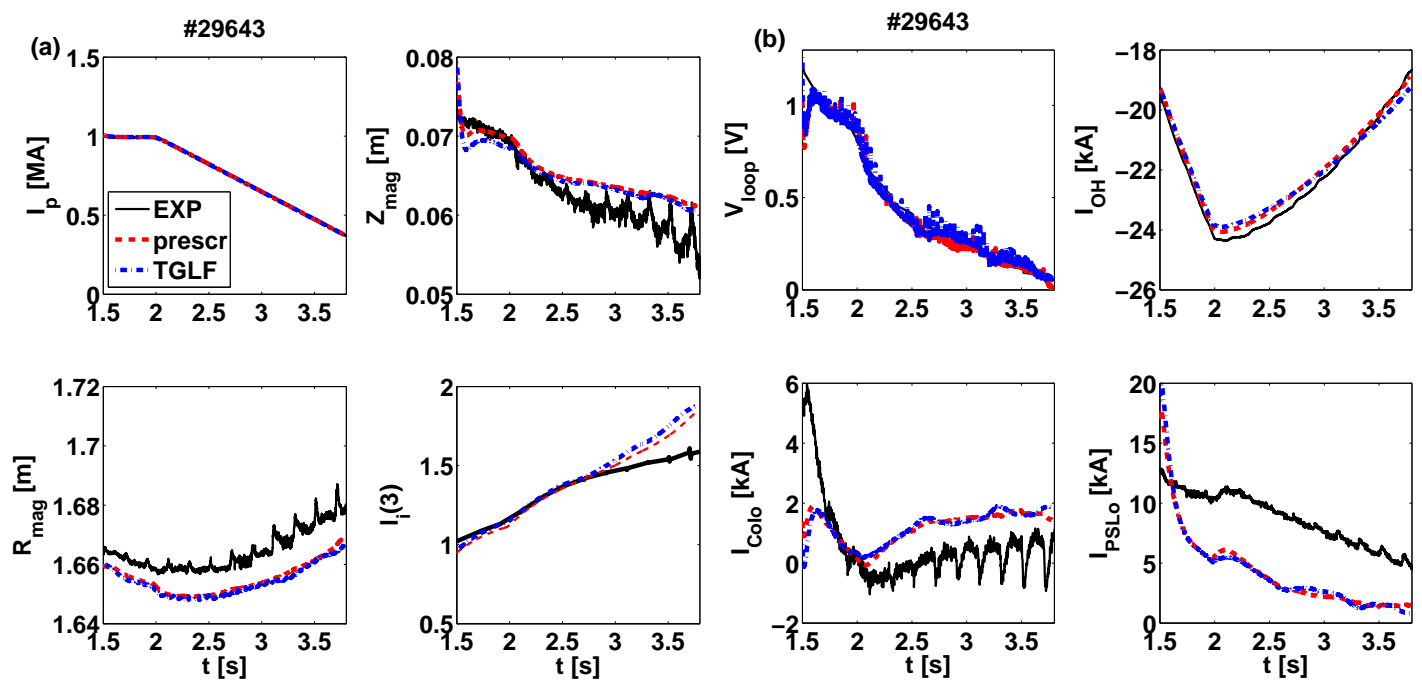

Figure 3. Same plot as in figure 1, but for ramp-down \#29643.
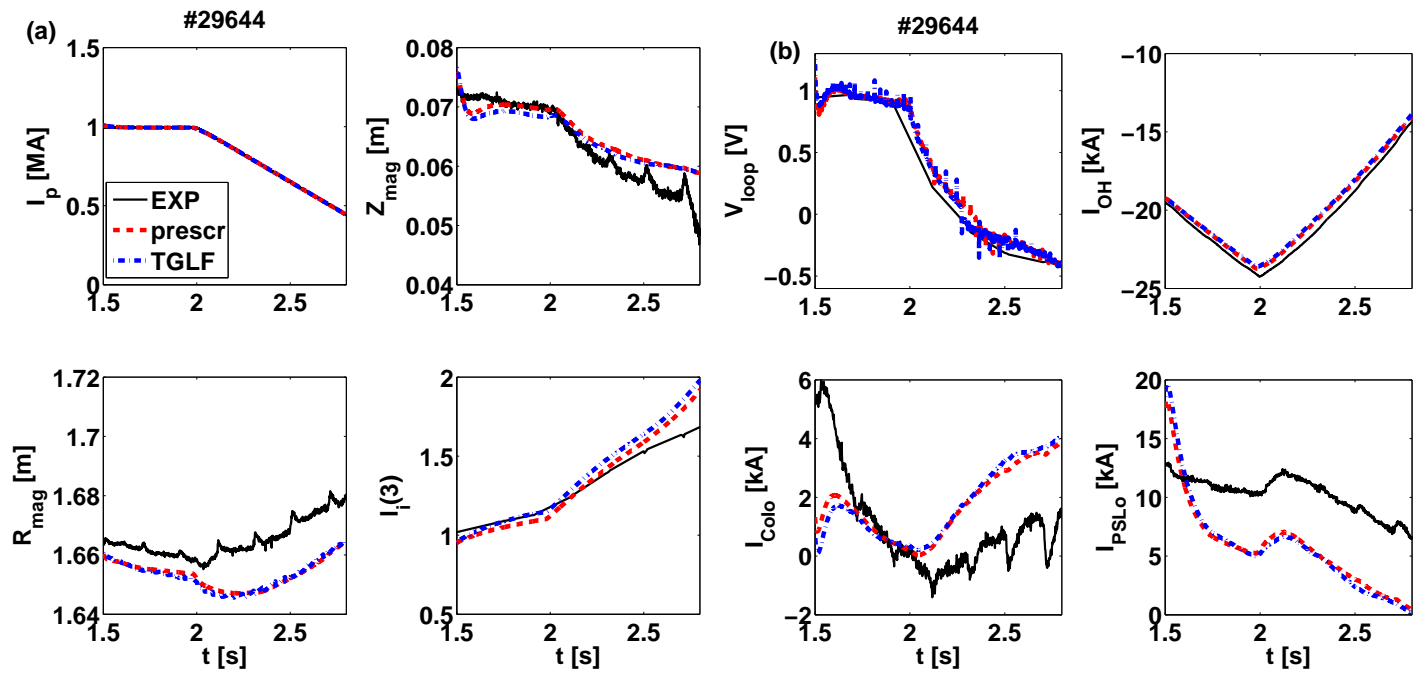

Figure 4. Same plot as in figure 1, but for ramp-down \#29644.

time traces (not shown) also have some discrepancy with respect to the measured one, possibly due to the simplified controller representation employed in this work. Despite this, it is remarkable that the internal inductance evolution is well reproduced in both cases, with the fastest ramp having the largest change in $l_{\mathrm{i}}(3)$, as expected. The TGLF modeling captures the main elements required for reproducing evolution of equilibrium and control parameters. Notice also that the NBI blips used for $T_{\mathrm{i}}$ measurements are not included in the simulation, which explains the model not being able to follow the periodic $\approx 200 \mathrm{~ms}$ oscillations observed in the time traces. 

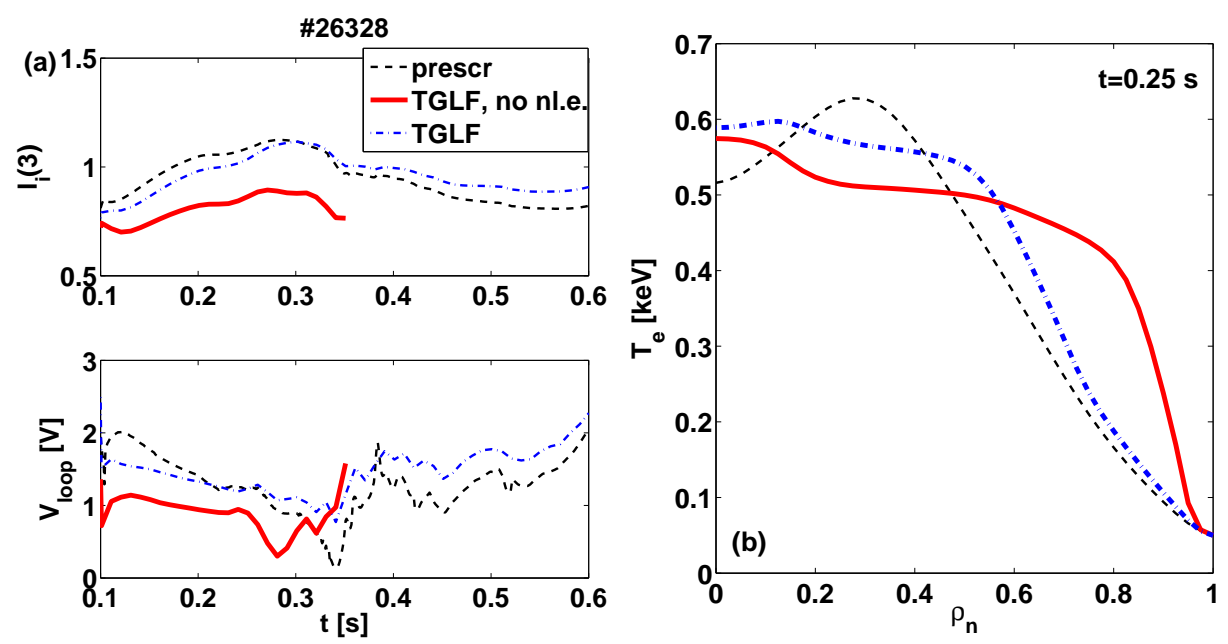

Figure 5. For ramp-up \#26328: a) time traces of $l_{\mathrm{i}}(3)$ (top) and of $V_{\text {loop }}$ [V] (bottom); b) $T_{\mathrm{e}}[\mathrm{keV}]$ radial profiles. Dashed line is experimental profile, solid line is TGLF modeling without enhancement, dot-dashed line is TGLF modeling with enhancement.

\subsection{TGLF+non-linear enhancement vs TGLF only}

A comparison is made between the simulations results using TGLF+non-linear enhancement and using TGLF only, to check the sensitivity of the evolution on the $T_{\mathrm{e}}$ shape resulting from the outer plasma transport properties, since this impacts strongly both $V_{\text {loop }}$ and $l_{\mathrm{i}}(3)$.

For the ramp-up \#26328 the result is shown in figure 5, while for the ramp-down \#29643 in figure 6. In figure 5(a) one clearly sees that TGLF without enhancement predicts a much lower $l_{\mathrm{i}}(3)$, as well as a lower $V_{\text {loop }}$. The reason is displayed in figure $5(\mathrm{~b})$, where the $T_{\mathrm{e}}$ profile is shown. Without enhancement (solid line), there is a strong increase of $T_{\mathrm{e}}$ near the edge, due to an underestimate of transport from TGLF, leading to the differences in global profiles mentioned before. Moreover, the simulation without enhancement crashes, since the plasma parameters at the transition between limited and diverted plasma (which happens at $t \approx 0.35 \mathrm{~s}$ ) lead to some numerical instability which does not happen when the enhancement is used.

In the case of the ramp-down, figure $6(\mathrm{a})$, global parameters are better matched even without enhancement, since in this case the $T_{\mathrm{e}}$ profile, shown in figure $6(\mathrm{~b})$ is not so different as in the ramp-up.

Notice that the free-boundary nature of the calculations allow to perform this sensitivity study of the kinetic profiles impact on the global magnetic quantities and thus on the controller, however the impact itself of the free-boundary evolution on the kinetic profiles is not dramatic, as an equivalent prescribed-boundary computation would leads to the same kinetic profiles. 

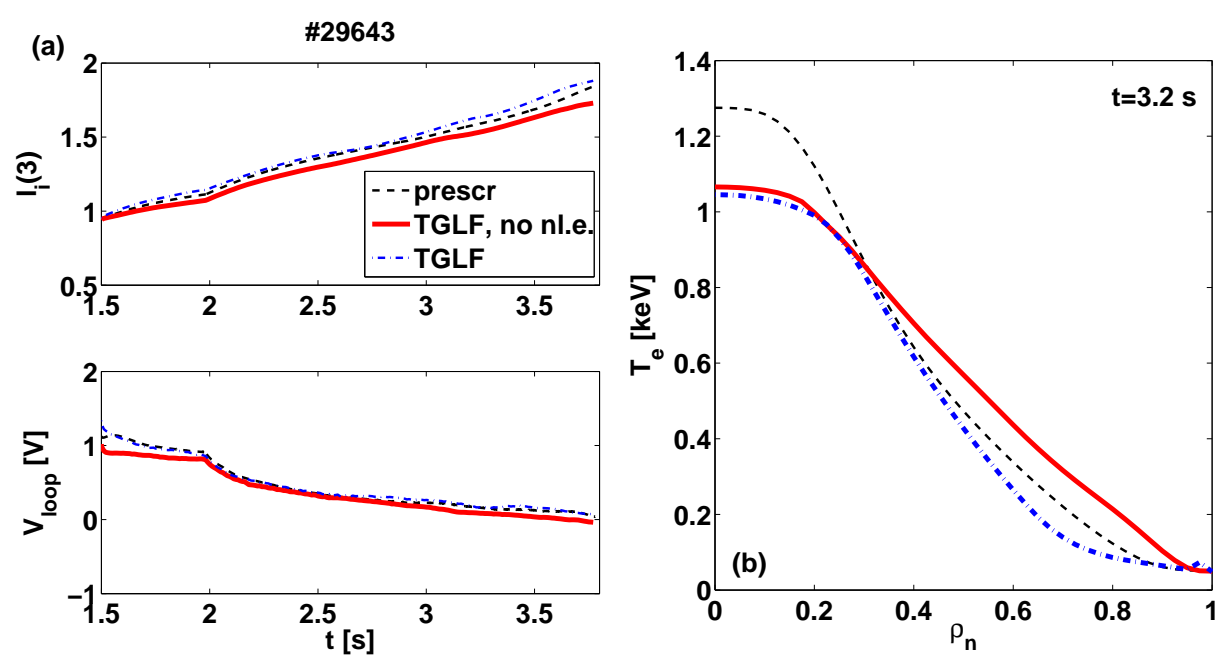

Figure 6. Same plot as in figure 5, but for ramp-down \#29643.
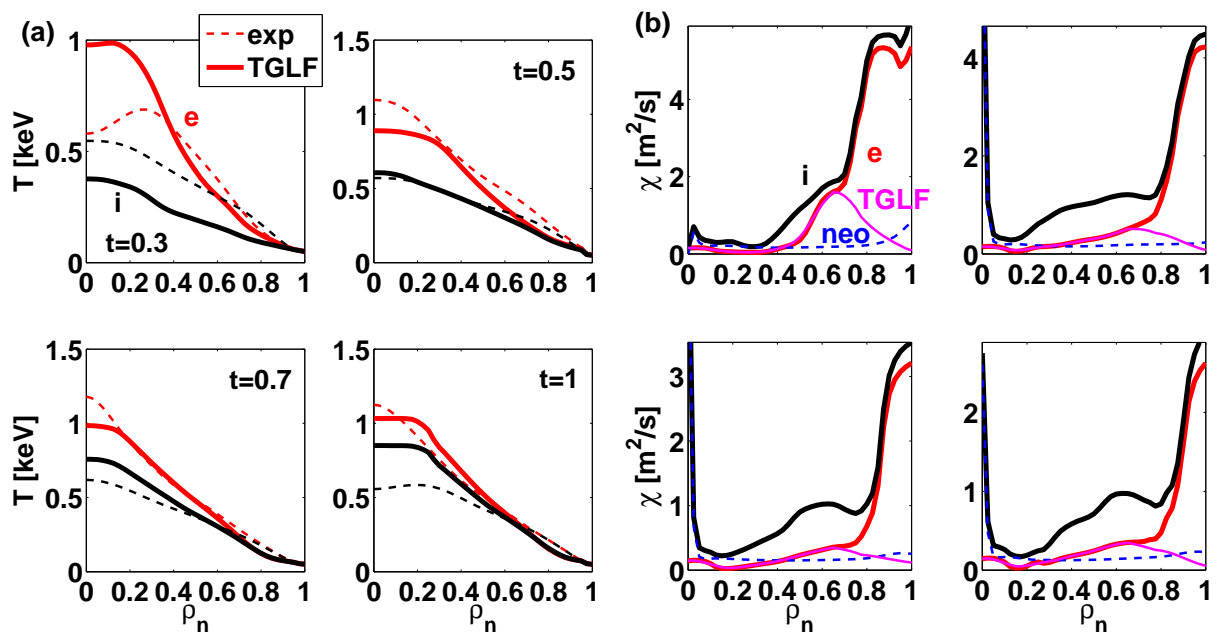

Figure 7. a) Profiles of $T_{\mathrm{e}}$ (e) and $T_{\mathrm{i}}$ (i) comparing experiment (dashed) and simulated (solid), for different time slices (time indicated in the plots); b) Profiles of heat diffusivities, also plotted neoclassical ion contribution (neo) and TGLF only (magenta, TGLF).

\subsection{Comparison of kinetic profiles and diffusivities}

To conclude this section on transport modeling, an example of how the $T_{\mathrm{e}}, T_{\mathrm{i}}$ profiles match between simulated and experiment is shown in figure 7(a), for four time slices of current ramp-up \#26328, using TGLF+non-linear enhancement. The two temperature profiles are well reproduced at all time slices, although, as previously discussed, the actual value of the non-linear enhancement which keeps the edge gradients moderate, could be re-tuned to get the best agreement with the internal inductance.

The heat diffusivities are plotted in figure $7(\mathrm{~b})$, where also the neoclassical contribution to ion heat diffusivity is shown separately, and the TGLF diffusivity profile 


\begin{tabular}{|c|c|c|c|c|}
\hline Case & $\begin{array}{c}0.8 \mathrm{MA} \\
r / a=0.75\end{array}$ & $\begin{array}{c}0.8 \mathrm{MA} \\
r / a=0.85\end{array}$ & $\begin{array}{c}0.4 \mathrm{MA} \\
r / a=0.75\end{array}$ & $\begin{array}{c}0.4 \mathrm{MA} \\
r / a=0.85\end{array}$ \\
\hline$R / L_{\mathrm{Te}}$ & 11.3 & 19 & 10.1 & 14.4 \\
\hline$R / L_{\mathrm{Ti}}$ & 8.1 & 11.3 & 5.6 & 6.6 \\
\hline$R / L_{\mathrm{n}}$ & 5.9 & 9.3 & 3.1 & 3.2 \\
\hline$q$ & 2.3 & 3.2 & 4.4 & 6 \\
\hline$\hat{s}$ & 2.3 & 3 & 2.3 & 2.9 \\
\hline$\hat{\mu}$ & 0.18 & 0.99 & 0.55 & 2. \\
\hline$\hat{\beta}$ & 0.3 & 0.88 & 0.96 & 2.34 \\
\hline
\end{tabular}

Table 1. Parameters for the gyro-kinetic computations for the four cases.

(for electrons) is drawn without the non-linear enhancement. It can be seen that the neoclassical contribution to ion heat transport is relevant only in the very core and at early times. The non-linear enhancement is far above TGLF in the outer part of the plasma, it is especially required at early times as a larger part of the plasma has a rather low diffusivity from TGLF.

\section{Transport analysis of outer plasma region for a plasma current scan}

The ability of the quasi-linear model to reproduce transport properties near the plasma edge is still something to be clarified, although from previous studies and present analysis of ramp scenarios it is clear that something is missing, most probably in the physics content. Previous studies seem to indicate a systematic underestimate when $q$ increases [22]. In some conditions also non-linear simulations seem to have pointed out this problem [30]. Recent efforts [31] show that cross-code comparison is needed to better understand this issue [32]. In gyro-kinetic simulations of Alcator C-Mod discharges, an underestimate of electron transport has been reported, which is attributed to ETG physics [33].

In this section, stationary phases of discharges \#28132 $\left(I_{\mathrm{p}}=400 \mathrm{kA}, q_{95}=12\right.$, $\left.P_{\mathrm{ECH}} \approx 1 \mathrm{MW}\right)$ and $\# 28151\left(I_{\mathrm{p}}=800 \mathrm{kA}, q_{95}=6, P_{\mathrm{ECH}} \approx 0.5 \mathrm{MW}\right)$ are studied with TGLF and with non-linear gyrokinetic codes GENE, GKW, to check if 1) transport fluxes are reproduced by either model, 2) what is the physics of transport as $q_{95}$ is increased.

While TGLF is ran over the whole radial domain, GENE and GKW are ran at two specific locations: $r / a \approx 0.75,0.85$, in flux-tube mode.

Relevant parameters for the four cases are shown in table 1. Note that, as discussed previously, that when $\hat{\mu}, \hat{\beta}$ are $\gtrsim 1$, one expects rather strong low- $n$ electro-magnetic activity competing with the usual TEM/ITG turbulence type (e.g. drift-waves [4], MTM [34]).

The gyro-kinetic codes run with pitch-angle+energy scattering collision operator 
(including conserving terms), electromagnetic effects (only $A_{\|}$included), and without equilibrium $\mathbf{E} \times \mathbf{B}$ shearing. Notice that the inclusion of $\mathbf{E} \times \mathbf{B}$ shearing leads in general to a reduction of non-linear fluxes, however for these kind of $\mathrm{L}$-mode, electron-heated plasmas, both the toroidal and poloidal D rotation are expected to be rather small, as well as the diamagnetic contribution. The radial electric field has in fact been measured via Doppler reflectometry in the radial region $r / a=[0.95-1]$, and at 0.95 it is already rather small in amplitude and flattish. As such, it is not expected that the shearing rate of the equilibrium electric field would play a dramatic role in these conditions; this in anyway left for future investigations. Two species (D and electrons) are used, with a finite $Z_{\text {eff }}$ that is included in the collision operator. The magnetic equilibrium is taken from SPIDER calculation. For TGLF, the Miller equilibrium parametrization is used.

First, a linear benchmark of the two gyro-kinetic codes GENE, GKW, and gyrofluid code TGLF has been performed and it is shown in figure 8(a) for the mode growth rate $\gamma$ and in figure $8(\mathrm{~b})$ for the mode real frequency $\omega$, both frequencies shown in units of $c_{\mathrm{s}} / a$. The agreement between the gyro-kinetic codes is extremely good for all cases. TGLF falls very close in terms of growth rate, while for the frequency there is some discrepancy in catching the TEM branch in some cases, and in giving the correct frequency in the high-k TEM branch.

From the $\omega$ plots one notices that in all these cases a mixture of TEM/ITG is present in the low $-k$ part of the spectrum. In these cases ETG modes are also found, however the spectrum is cut in the non-linear simulations to exclude the ETG contribution (left for future studies). At the very low-k, the gyrokinetic results show also some modes rotating in the electron diamagnetic directions. These have been identified as microtearing mode, and they could play a role in the non-linear dynamics [34]. Notice how they tend to occupy a larger portion of the low $-\mathrm{k}$ spectrum as $\hat{\beta}$ is increased going from high-current to low-current case.

The non-linear simulation results are shown in figure 9(a) for the high current case and in figure 9(b) for the low current case. The power balance heat flux in MW is compared to the GENE/GKW simulations and to TGLF quasi-linear results. Variation bounds for power balance heat flux due to uncertainties in radiated power is given as an error bar. Notice that the convective contribution (i.e. due to a finite particle flux) has been subtracted to get the purely conductive heat fluxes. In addition, the neoclassical contribution to the ion heat flux has also been subtracted from the power balance calculation.

For the high current case, figure 9(a), the agreement between GENE, GKW, and TGLF with respect to experimental profiles is very good. For this case, it looks like no additional physics is required to explain the experimental profile, either from quasilinear modeling (TGLF) or from non-linear, flux-tube calculations (GENE/GKW).

For the low current case, figure $9(\mathrm{~b})$, the calculation at $r / a=0.75$ is also close to experimental value, for the non-linear computations, while TGLF underestimates the fluxes by a factor $\sim 4$.

To understand where the discrepancy between the non-linear calculations and 

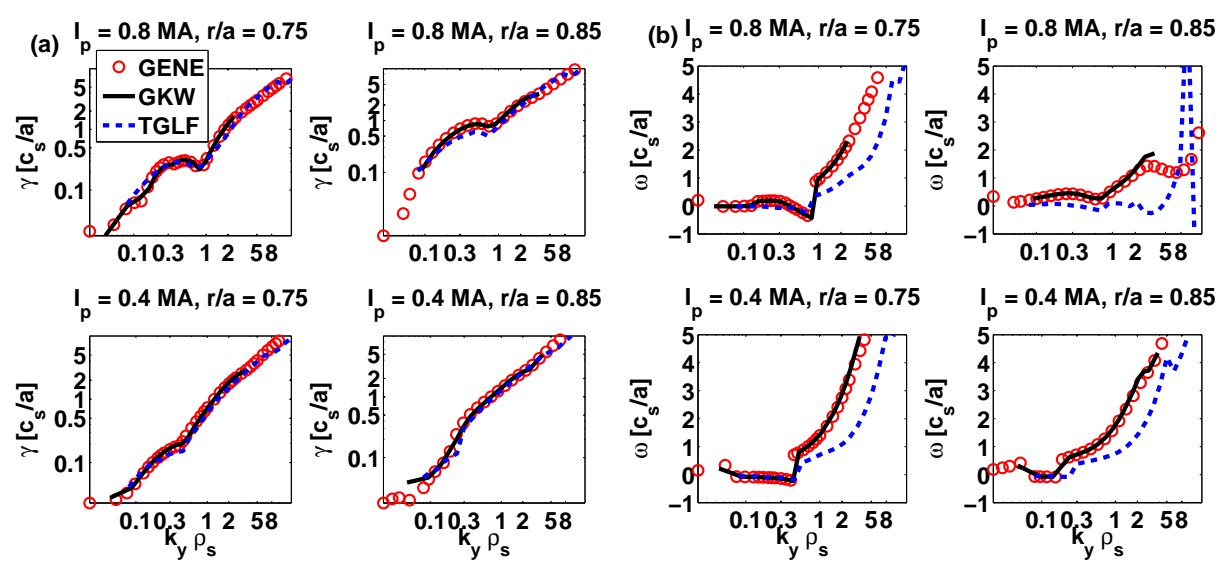

Figure 8. Comparison of linear spectra for the a) mode growth rate $\gamma$ and b) mode real frequency $\omega$ for the different current cases and radial positions (in the titles). Frequencies are in units of $c_{\mathrm{s}} / a$. GENE is in red dots, GKW is in solid black lines, and TGLF is in dashed blue lins. Positive frequency indicates rotation in the electron diamagnetic direction.
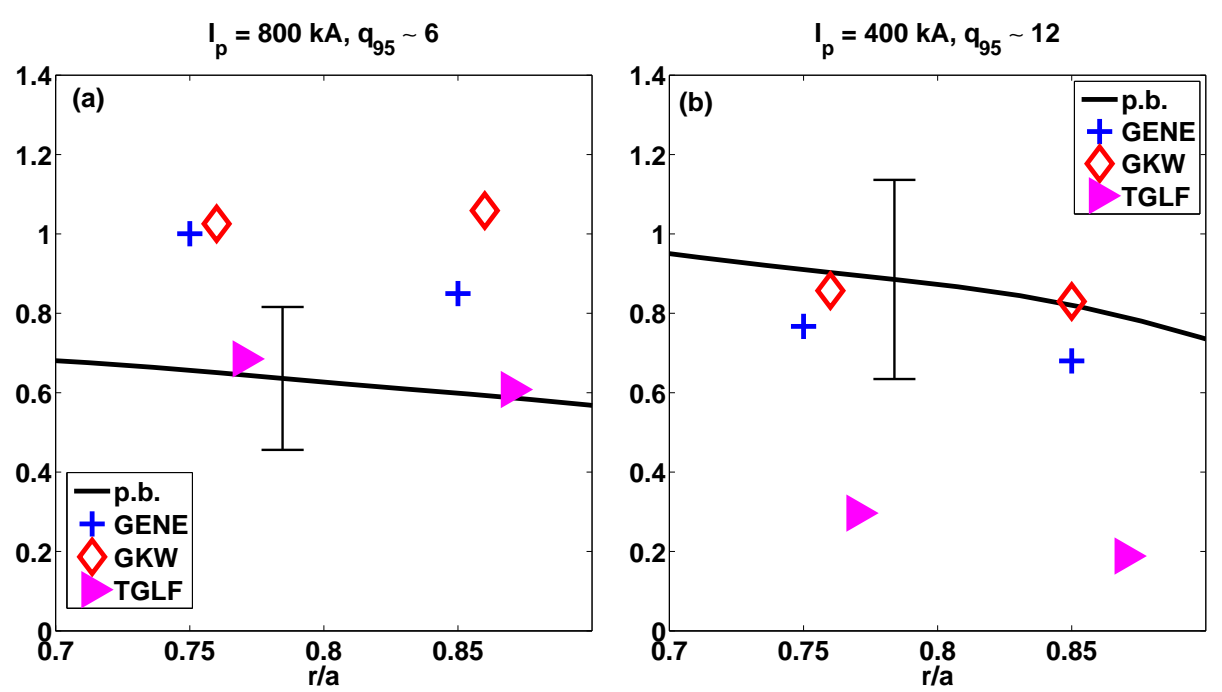

Figure 9. Comparison of heat fluxes in MW: from power balance (black), GENE (crosses), GKW (diamonds), and TGLF (triangles). The error bar on the power balance is due to uncertainties in radiated power. a) high current case; b) low current case.

the quasi-linear result could come from for the low-current case, in figure 10(a) the spectrum of linear cross-phase between electron pressure fluctuation and electrostatic potential is compared between GKW and TGLF. To build a quasi-linear flux from linear calculations, this is one fundamental piece of information, the other one being the growth rate plus some other less important quantitites. So, comparing the linear growth rates and the cross-phase, it looks like that TGLF should behave in the same way as 

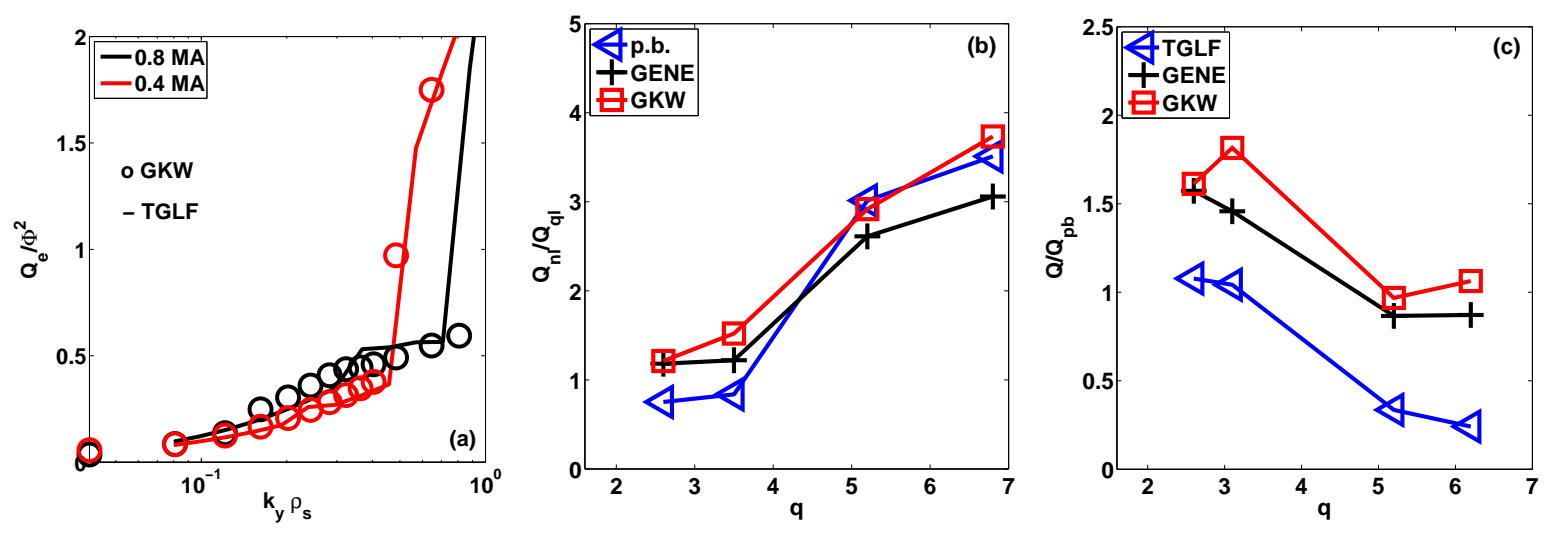

Figure 10. a) Linear spectra of electron energy phase-shift: GKW (circles) vs TGLF (lines) for the high current (black) and low current (red) case at $r / a=0.75$; b) Ratio of power balance (p.b.) and non-linear heat-flux (GENE/GKW) versus quasi-linear (TGLF) heat fluxes as a function of the local safety factor (combines low and high current at both radii); c) Same as b), but the ratio is between GENE, GKW, TGLF fluxes over the power-balance flux.

GKW in terms of "quasi-linear" fluxes. However, GKW computes a non-linear flux in the low-current case that is a factor $\approx 3$ larger than TGLF, as shown in figure 10(b). GENE gives the same result. To put in evidence the difference between non-linear and quasi-linear prediction over power-balance, the ratio is shown in figure 10(c), where it can be seen that, at low values of the safety factor, GENE/GKW are above TGLF by a factor $\approx 1.5$, while in the case of high values of safety factor, this ratio becomes $\approx 3-4$. The non-linear turbulence state seems to differ rather strongly from the quasi-linear expectation in the low-current case. At this point three possible explanations can be offered for this: 1) the quasi-linear rule itself needs to be retuned considering a larger parameter space (high $-q$, high- $\hat{\beta}$ ); 2 ) energy redistribution in t the spectrum when going to low-current, e.g. low-k condensation or other effects not yet studied in detail, would require re-tuning of the model spectrum according to more non-linear simulations; 3 ) the non-linear state of turbulence cannot be captured by a quasi-linear model with a fixed $Q / \gamma$ scaling. It looks like this issue is not yet solved, however it looks from the present simulations performed in this work that local non-linear gyro-kinetic simulations with standard physics ingredients are enough to recover experimentally sizeable fluxes.

\section{Conclusions}

This work has presented validation of a novel free-boundary transport package composed of ASTRA+SPIDER+additional transport modules (specifically TGLF for kinetic profiles transport), plus analysis of the 'short-fall' problem, relevant for low current scenarios, with gyro-kinetic codes.

The simulations of the current ramps scenarios show very good agreement between 
the code and the experiment, despite many uncertainties on several key parameters, and the employment of a simplified controller model. The TGLF+non-linear enhancement simulations follow very closely the cases with prescribed kinetic profiles. These results give confidence that TGLF+non-linear enhancement can be a reliable theory-based tool (which finalization requires a better physical understanding on how to theoretically predict the enhancement) for full-discharge modeling.

Non-linear gyrokinetic computations of two stationary discharges at different $q_{95}$ show that good agreement is obtained for the low $q_{95}$ case, where no short-fall is observed in both ions and electrons channels. For the high $q_{95}$ there is good agreement, although further investigations in the interplay between the TEM/ITG modes and low-n electromagnetic modes are required.

The present work motivates application of the numerical package to more scenarios and to foreseen machines (e.g. ITER, DEMO). Several issues have to be investigated and ameliorated in future work, in particular: controller representation, theory-based definition of the core-edge enhancement according to non-linear results, and possible integration of a SOL model. The latter is at the moment completely missing with respect to halo currents for example, while for SOL transport in principle the STRAHL model is already implemented [35], and SOLPS model has been started to be integrated in ASTRA [36]. Of course adding SOL physics will make the computation very timeconsuming, something that will require thinking on how to focus the package if, for example, a flight-simulator would be likely used. Extending the simulations capability to the breakdown phase of the plasma is also planned.

\section{Acknowledgments}

Useful discussions with Dr. B. D. Scott are acknowledged. The gyrokinetic and transport simulations have been carried out on the Linux cluster at the RZG center in Garching b. Muenchen, and on the HELIOS super-computer at Rokkasho, Japan.

\section{References}

[1] G. V. Pereverzev et al., IPP Report 5/42 (August 1991)

[2] A. A. Ivanov et al., 32nd EPS Conf. on Plasma Phys., ECA Vol.29C, P-5.063 (2005), link: http://epsppd.epfl.ch/Tarragona/pdf/P5_063.pdf

[3] G. M. Staebler et al., Phys. Plasmas 14, 055909 (2007)

[4] B. D. Scott, Phys. Plasmas 7, 1845 (2000)

[5] G. Bracco et al., 7th EU-US Transport Task Force Workshop,Varenna, Italy, September 4-7, (2000), link: http://www.afs.enea.it/bracco/papers/TTF2000_Varenna_Bracco.pdf

[6] G. L. Jackson et al., Phys. Plasmas 17, 056116 (2010)

[7] P. A. Politzer et al., Nucl. Fusion 50, 035011 (2010)

[8] I. Voitsekhovitch et al., Plasma Phys. Control. Fusion 52, 105011 (2010)

[9] G. Granucci et al., Nucl. Fusion 51, 073042 (2011)

[10] F. Imbeaux et al., Nucl. Fusion 51, 083026 (2011)

[11] Y. Gribov et al., Nucl. Fusion 47, S385 (2007)

[12] S. H. Kim et al., Plasma Phys. Control. Fusion 51, 065020 (2009)

[13] E. Fable et al., Nucl. Fusion 51, 043006 (2011) 
[14] E. Fable et al., Nucl. Fusion 52, 063017 (2012)

[15] S. Fietz et al., Nucl. Fusion 53, 053004 (2013)

[16] F. Jenko et al., Phys. Plasmas 7, 1904 (2000)

[17] A. G. Peeters et al., Comput. Phys. Commun. 180, 2650 (2009)

[18] E. Fable et al., Plasma Phys. Control. Fusion 55, 074007 (2013)

[19] W. Treutterer et al., Fusion Eng. and Design 74, 501 (2005)

[20] J. E. Kinsey et al., Phys. Plasmas 15, 055908 (2008)

[21] F. Sommer et al., RF conference in Sorrento (2013)

[22] G. M. Staebler et al., Overview of turbulence and transport in the core-edge transition region, presented at the EU-US TTF, 3-6 September, Padova (2012)

[23] W. A. Houlberg et al., Phys. Plasmas 4, 3230 (1997)

[24] O. Sauter et al., Phys. Plasmas 6, 2834 (1999)

[25] F Porcelli et al., Plasma Phys. Control. Fusion 38, 2163 (1996)

[26] E. Poli et al., Comput. Phys. Commun. 136, 90 (2001)

[27] R. Fischer et al., Fus. Sci. and Tech. 58, 675 (2010)

[28] P. J. McCarthy et al., Plasma Phys. Control. Fusion 54, 015010 (2012)

[29] S. K. Rathgeber et al., Plasma Phys. Control. Fusion 52, 095008 (2010)

[30] C. Holland et al., Phys. Plasmas 18, 056113 (2011)

[31] F. Jenko et al., ITPA meeting on transport and confinement, Garching bei München (2013)

[32] R. V. Bravenec et al., Phys. Plasmas 18, 122505 (2011)

[33] N. T. Howard et al., Phys. Plasmas 20, 032510 (2013)

[34] H. Doerk et al., Phys. Plasmas 19, 055907 (2012)

[35] R. Dux, IPP report 10/30 (2006)

[36] I. Yu. Senichenkov et al., 38th EPS Conference on Plasma Physics (2011) P5.115, link: http://ocs.ciemat.es/EPS2011PAP/pdf/P5.115.pdf 